\title{
Critical Exponents and Elementary Particles
}

\section{J. Glimm}

The Rockefeller University, New York, NY 10021, USA

A. Jaffe ${ }^{\star \star}$

Harvard University, Cambridge, MA 02138, USA

\begin{abstract}
Particles are shown to exist for a.e. value of the mass in single phase $\phi^{4}$ lattice and continuum field theories and nearest neighbor Ising models. The particles occur in the form of poles at imaginary (Minkowski) momenta of the Fourier transformed two point function. The new inequality $d^{2} / d \sigma \leqq Z$, where $\sigma=m_{0}^{2}$ is a bare mass ${ }^{2}$ and $Z$ is the strength of the particle pole, is basic to our method. This inequality implies inequalities for critical exponents.
\end{abstract}

\section{Introduction}

Euclidean $\phi^{4}$ fields are believed to describe the asymptotic long distance behavior of certain lattice models of statistical mechanics at their critical points (e.g. the Ising model). It was proposed [9] that a construction of $\phi^{4}$ fields could be based on this expectation, and partial results in this direction are given in $[6-8,10,11,1,12]$. In this construction, the field $\phi$ will be nontrivial (i.e. not a free field) only in the case in which the corresponding lattice model critical point is asymptotically nontrivial at long distances.

In order to better distinguish between the trivial and the nontrivial cases, we continue here our investigation $[7,10,11]$ of critical exponents (see also $\S 5$ ). In general, our results have the form

$$
\begin{aligned}
& \text { canonical exponent } \leqq \text { general exponent } \\
& \phi \text {-exponent } \leqq \phi^{2} \text {-exponent, }
\end{aligned}
$$

and in particular if the lattice $\phi^{2}$ field (e.g. the Ising model energy-energy correlation) is canonical, then so is the corresponding lattice $\phi$-field and also the resulting continuum $\phi$-field. The converse to this statement seems to be false, and a counterexample may be found in the $\phi_{4}^{4}$ lattice field at weak coupling. In this model, there is some evidence that $\phi^{2}$ deviates from canonical by a logarithm.

* Supported in part by the National Science Foundation under grant PHY 76-17191

$\star \star$ Supported in part by the National Science Foundation under grant MPS 75-21212 
The bounds (1.1) are given in [7] while the bound [10]

$$
\eta \leqq \eta_{E} / 2
$$

of the form (1.2) is an elementary consequence of Lebowitz' inequality. This inequality compares the anomalous dimension $\eta$ for $\phi$ with the anomalous dimension $\eta_{E}$ for $\phi^{2}$. One result of this paper is an improvement on the bound [3] $1 \leqq(2-\eta) v$, namely

$$
1 \leqq(2-\zeta / v) v
$$

where $v$ is the exponent for the mass and $\zeta$ the exponent for field strength renormalization. (Note $\zeta / v \geqq \eta$ in the case of Euclidean covariance and $\zeta / v=\eta$, assuming scale relations.) The stronger upper bounds on $\eta$ in [11] required stronger hypothesis.

Our proof of (1.4) follows from a stronger inequality for an Ising model, lattice $\phi^{4}$ fields or $d=1,2,3$ continuum $\phi^{4}$ fields. We show that

$$
d m^{2} / d \sigma \leqq Z, \text { assuming } \sigma>\sigma_{c}
$$

where $Z$ is the strength of the one particle pole in the truncated two point function, $m^{-1}$ is the correlation length and $\sigma$ is the bare mass ${ }^{2}$ (or inverse temperature). The equality (1.5) improves $d m^{2} / d \sigma \leqq 1$, established in [7], and integration of (1.5) yields the relation (1.4) for critical exponents. (Recall that $m \searrow 0$ as $\sigma \searrow \sigma_{c}[1,11]$.)

The main result of the present paper is that $d m^{2} / d \sigma \neq 0$ for almost all masses $m$, so that by (1.5), $Z \neq 0$ for almost all masses. We infer that an elementary particle (a pole in the propagator) exists for almost all masses in the region $\sigma>\sigma_{c}$, i.e. the single phase region. Also as $\sigma \searrow \sigma_{c}$,

$$
\chi=\int G^{(2)}(x) d x \rightarrow \infty,
$$

and so

$$
\Gamma^{(2)}(p=0) \rightarrow 0 \quad \text { as } \quad \sigma \downarrow \sigma_{c} .
$$

This convergence was assumed in [7] in the derivation of the inequality $\gamma \geqq 1$. We give proofs for continuum fields, and the same methods then carry over to lattice fields and nearest neighbor Ising models on a rectangular lattice. For the Ising models $\sigma$ (the coefficient of $\phi^{2}$ in the $\phi^{4}$ interaction) is replaced by $-\beta$.

\section{Critical Behavior of the Mass and the Field Strength Renormalization}

For pure imaginary (Minkowski) momentum ip, $p$ real, we define

$$
\chi(p)=\int G(x) e^{-p x} d x=G(i p)
$$

with $\int \ldots d x$ replaced by a summation in the lattice case. We choose ip inside the tube of analytically of $G^{\sim}$. Then

$$
\begin{aligned}
0 & \leqq-d \chi(p) / d \sigma=\frac{1}{2} \int\left\langle\phi(x): \phi(y)^{2}: \phi(0)\right\rangle e^{-p(x-y)} e^{-p y} d x d y \\
& \leqq \int\langle\phi(x) \phi(y)\rangle\langle\phi(y) \phi(0)\rangle e^{-p(x-y)} e^{-p y} d x d y=\chi(p)^{2}
\end{aligned}
$$


Thus

$$
0 \leqq-d \Gamma / d \sigma=-d(-\chi(p))^{-1} / d \sigma=-\chi^{-2} d \chi(p) / d \sigma \leqq 1
$$

for all such $p$. Since we have used translation invariance, the proof applies in the infinite volume case, and also in the finite volume periodic case.

To apply this inequality in a finite volume with periodic boundary conditions, we note that the Hamiltonian has discrete spectrum, and that its first excited state occurs in the spectral decomposition of the two point function. Furthermore, the first excited state occurs at zero momentum. Thus we take $p=\left(p_{0}, \boldsymbol{p}\right)$, with $\boldsymbol{p}=0$, and $Z^{-1}<\infty$ for all volumes $V<\infty$. Let $m_{p, V}$ be the periodic mass. For $-p_{0}^{2}<m^{2}, \Gamma$ is an analytic function of the independent variables $\sigma$ and $p_{0}^{2}$ by a standard application of the Paley-Wiener theorem, cf. $[11, \S 2]$. The one particle curve $+p_{0}^{2}=m^{2}$ is analytic in any region free of level crossings in the first excited state, and thus is at least piecewise analytic. $[15, \mathrm{VII}, \S 1]$ can be applied to the Hamiltonian $H(\sigma)$, which is analytic in $\sigma$ by second order estimates [16]. For example the inequality $H(\operatorname{Re} \sigma)^{2}$ $\leqq$ const $H\left(\sigma^{-}\right) H(\sigma)+$ const implies that $H(\sigma)$ is closed, on the domain $\mathscr{D}(H(\operatorname{Re} \sigma))$. With the same discrete exceptions at possible level crossings, $\Gamma$ vanishes along the one particle curve $p^{2}=-m(\sigma)^{2}$. From these facts, it follows $d \Gamma / d p^{2}$ is a multiple of $d \Gamma / d \sigma$ on this one particle curve. In particular, in the $\left(\sigma,-p^{2}\right)$ plane, the vector $\left(1, d m^{2} / d \sigma\right)$ lies along the one particle curve, and so

$$
0=\nabla \Gamma \cdot\left(d m^{2} / d \sigma, 1\right)=\left[\partial \Gamma / \partial\left(-p^{2}\right)\right] d m^{2} / d \sigma+\partial \Gamma / d \sigma
$$

or

$$
0 \leqq Z^{-1}=-\partial \Gamma /\left.\partial p^{2}\right|_{p^{2}=-m^{2}}=-[\partial \Gamma / \partial \sigma]\left[d m^{2} / d \sigma\right]^{-1} .
$$

Thus by (2.1),

$$
0 \leqq d m^{2} / d \sigma=-Z \partial \Gamma / \partial \sigma \leqq Z,
$$

proving (2.3). In the case of level crossings, one sided derivatives $d \mathrm{~m}^{2} / d \sigma^{ \pm}$satisfy (2.3), since $Z$ is semicontinuous at a level crossing.

For later use, we note that

$d m^{2} / d \sigma \leqq$ const.

by [7] in the continuum case, or (2.3), since $Z \leqq 1$ for canonical continuum fields. For lattice fields and Ising models, the $p$-space canonical upper bound [4] on the two point function, and its Herglotz representation [11], show $Z \leqq$ const. Thus by (1.6), the bound (2.4) extends to $\phi^{4}$ lattice fields and Ising models in a finite periodic volume, with a constant independent of the volume.

By passing to the subsequence $V_{j} \rightarrow \infty$, we have $m_{p, V} \rightarrow m_{p, \infty}$ but we do not know (yet) that $m_{p, \infty}$ is the mass $m_{p}$ of the corresponding infinite volume theory. However, by semicontinuity of the spectrum.

$$
m_{p, \infty} \leqq m_{p}
$$

By [5], $m_{p}=m_{d}$, where $m_{d}$ is the mass in a theory with Dirichlet boundary conditions. We define the corresponding critical values $\sigma_{c d}=\sigma_{c p} \leqq \sigma_{c, p, \infty}$. By (2.4) and Ascoli's theorem, we have uniform convergence and uniform Lipschitz 
continuity of $m_{p, V}^{2}(\sigma)$ and the limit $m_{p, \infty}^{2}(\sigma)$. In particular, both $m_{d}$ and $m_{p \infty}$ go continuously to zero at their critical points $[7,1,12]$.

By definition of $\sigma_{c}$, the quantity $d m^{2} / d \sigma$ cannot vanish in a one-sided neighborhood $\left(\sigma_{c}, \sigma_{c}+\varepsilon\right]$ of $\sigma_{c}$, and by (1.5) the measure of the set on which $\mathrm{dm}^{2} / d \sigma$ $\neq 0$ is proportional to $m^{2}\left(\sigma_{c}+\varepsilon\right)$, uniformly in $V$. It follows that by the diagonal process, we can choose $V_{j} \rightarrow \infty$ so that there is a sequence $\sigma_{i} \downarrow \sigma_{c p \infty}$ with the strict inequality

$$
\lim _{V_{j} \rightarrow \infty} d m^{2} /\left.d \sigma\right|_{\sigma=\sigma_{i}}>0 \text {. }
$$

For these values of $\sigma_{i}$, we also have $Z$ bounded away from zero by (1.5), which implies $m_{p}=m_{p \infty}$ for $\sigma=\sigma_{i}$. Because the $m$ 's are monotone in $\sigma$, it follows that $\sigma_{c d}$ $=\sigma_{c p}=\sigma_{c p \infty}$.

Extending the above argument, we can work with $\Sigma=\left\{\sigma: d m_{p \infty}^{2} / d \sigma>0\right\}$, which is a set of positive measure in every interval $\left(\sigma_{c}, \sigma_{c}+\varepsilon\right)$. On this set $Z \neq 0$ and $m_{p \infty}=m_{p}$. However since $m_{p \infty}$ is constant on $\sim \Sigma$, while $m_{p}$ is monotone (and hence monotone on $\Sigma$ ) we must have $m_{p \infty}=m_{p}$ everywhere. We summarize these results.

Theorem 1. For $\sigma>\sigma_{c}$, we have

$$
m_{p \infty}=m_{p}=m_{d}
$$

and

$$
d m^{2} / d \sigma \leqq Z(\sigma) .
$$

Furthermore $Z(\sigma)$ uppersemicontinuous :

$$
Z(\sigma) \leqq \lim \sup _{\sigma^{\prime} \rightarrow \sigma} Z\left(\sigma^{\prime}\right)
$$

We now argue that $Z \neq 0$ for all masses $m$ not in a set of measure zero. Consider $m^{2}(\sigma)$ and $\sigma\left(m^{2}\right)$ as monotone functions. Thus they have bounded variation and are differentiable a.e. with derivatives $m^{2 \prime}, \sigma^{\prime}$ which are positive and $L_{1}$. Thus

$$
\left(\sigma^{\prime}\right)^{-1}=d m^{2} / d \sigma \neq 0 \text { a.e. }
$$

as a function of $m$, and by (2.3) $Z \neq 0$ a.e. as a function of $m$. The region of non uniqueness of mass renormalization corresponds to intervals in which $\mathrm{m}^{2}(\sigma)$ $=$ const., hence to $\delta$-functions in the measures $d \sigma\left(\mathrm{m}^{2}\right)$. These occur for a most countable number of values of $\mathrm{m}^{2}$.

Remark. The above result completes the proof of the second of three steps of [9] for a possible construction ${ }^{1}$ of $\Phi_{d}^{4}$ fields : that defined by the long distance behavior of the corresponding lattice field at its critical point. The first step was established in $[7,1,12]$. The third step seems to depend on showing scaling behavior as $\sigma \searrow \sigma_{c}$, i.e. the isolation of leading long distance behavior from next to leading, etc.

\section{Divergence of $\chi$ at the Critical Point}

In this section we show that $\chi_{c}=\chi\left(\sigma_{c}\right)=\infty$, which is related to the upper bound $\eta \leqq 2$. Again we work in the $\left(\sigma,-p^{2}\right)$ parameter space, but in the $V=\infty$ theory directly. Below or on the one particle curve $-p^{2}=m^{2}$, the quantity $\partial \Gamma / \partial \sigma$ is bounded, while

$1 \quad$ If the nontrivial part of the critical behavior occurs only in the next to leading terms for all values of the bare charge, then the construction of [9] is inappropriate (cf. $\S 5$ ) 
below this curve or on nonhorizontal portions of this curve, $\partial \Gamma / \partial\left(p^{2}\right)$ is bounded also, and $\Gamma$ vanishes, at least on non-horizontal portions of the curve. Moreover, by the Herglotz representation for $\Gamma$, any bound on $\partial \Gamma / \partial\left(-p^{2}\right)$, with $p^{2}=-m^{2}$, extends to $0 \leqq-p^{2} \leqq m^{2}$, since $\partial \Gamma / \partial\left(-p^{2}\right)$ is decreasing in $-p^{2}$ in this interval. Thus by (2.2) and integration,

$$
0 \leqq-\Gamma\left(p^{2}=0, \sigma=\sigma_{i}\right) \leqq 0\left(m^{2}\right)\left(d m^{2} / d \sigma\right)^{-1}
$$

where $\sigma_{i}$ is chosen as in $\S 2$, so that $Z \neq 0$, hence $G\left(p^{2}=-m^{2}\right)=\infty$ and $\Gamma\left(p^{2}=-m\right)$ $=0$. Thus

$$
0(1) m^{-2} d m^{2} / d \sigma \leqq \chi_{\sigma_{l}} \text {. }
$$

Assuming $\chi_{\sigma_{c}}$ finite, we have

$d m / d \sigma \leqq$ const. $m$.

Recall that $m\left(\sigma_{c}+0\right)=0$, so that after integration from $\sigma_{c}$ to $\sigma>\sigma_{c}$,

$$
0 \leqq m(\sigma) \leqq m\left(\sigma_{c}+0\right) e^{\text {const. }\left(\sigma-\sigma_{c}\right)}=0 .
$$

This is a contradiction, which shows that $\chi_{c}=\infty$. As a corollary of this result, we observe that $\sigma_{c}$ is characterized as the largest value of $\sigma$ for which the effective potential (see [2]) has zero curvature at the origin:

$$
\left.V^{\prime \prime}\right|_{\sigma=\sigma_{c}}=\left.\Gamma^{(2)}(p=0)\right|_{\sigma=\sigma_{c}}=0 \text {. }
$$

\section{The Ising Limit}

We consider the interactions

$$
\frac{1}{2} \sum_{n . n}\left(\phi_{i}-\phi_{i^{\prime}}\right)^{2}+\sum_{i}\left[\lambda\left(\phi_{i}^{2}-1\right)^{2}+\frac{1}{2} \sigma \phi_{i}^{2}\right]
$$

and

$$
-\sum_{n . n} \beta \psi_{i} \psi_{i^{\prime}}+\sum_{i} \lambda_{0}\left(\psi_{i}^{2}-1\right)^{2}
$$

where $\sum_{n . n}$ denotes a sum over nearest neighbor pairs. With

$$
\psi=(1-(\sigma+2 d) / 4 \lambda)^{-1 / 2} \phi
$$

and

$$
\lambda_{0}=\lambda-(\sigma+2 d) / 4 \lambda, \quad \beta=1-(\sigma+2 d) / 4 \lambda,
$$

these two expressions differ by a constant, and thus define the same lattice field theory. For the purpose of studying the Ising limit ( $\lambda$ or $\lambda_{0} \rightarrow \infty, \beta$ fixed), it is convenient to use the representation (4.2). Let $\beta_{c I}$ be the critical value of $\beta$ in the Ising model.

Theorem 2. As $\lambda_{0} \rightarrow \infty, m\left(\beta, \lambda_{0}\right) \rightarrow m_{I}(\beta)$, the Ising model mass, for $\beta<\beta_{c, I}$, and $\beta_{c, I}$ $\leqq \lim _{\lambda_{0} \rightarrow \infty} \beta_{c}\left(\lambda_{0}\right)$.

Proof. As in $(2.1),-d \Gamma / d(-\beta) \leqq 2$ for imaginary momenta below the one particle curve, and as in (2.3), $0 \leqq d m^{2} / d(-\beta) \leqq 2 Z$. These bounds are uniform in $\lambda_{0} \leqq \infty$, in the one phase region. Thus they imply uniform convergence (through a subsequence 
$\left.\lambda_{0 j} \rightarrow \infty\right)$ and uniform Lipschitz continuity in $\beta$ of $m^{2}\left(\beta, \lambda_{0}\right)$. Let $m^{2}(\beta, \infty)$ be the limit value of the mass ${ }^{2}$, and let $m_{I}^{2}(\beta)$ be the Ising model mass ${ }^{2}$. We have $m^{2}(\beta, \infty) \leqq m_{I}^{2}(\beta)$ for $\beta \geqq \beta_{c, I}$ by semicontinuity of the spectrum. Next we choose values $\beta_{i}$ for which $Z\left(\beta_{i}, \lambda_{0}\right)$ is bounded away from zero as $\lambda_{0, j} \rightarrow \infty$. For these values of $\beta_{i}$, we have $m^{2}(\beta, \infty)=m_{I}^{2}(\beta)$. By monotonicity, $m^{2}(\beta, \infty)=m_{I}^{2}(\beta)$ throughout the single phase region. Convergence of the full sequence follows from uniqueness of the limit, in the single phase region. With convergence of the masses assured for $\beta \leqq \beta_{c, I}$, it follows that $\lim _{\lambda_{0} \rightarrow \infty} \beta_{c}\left(\lambda_{0}\right) \geqq \beta_{c, I}$.

The same method applies to variation of the $\beta$ 's which couple between layers. Let $J=\left(J_{1}, \ldots, \mathrm{J}_{d}\right)$ and let

$$
H(\sigma)=-\frac{1}{2} \Sigma \beta J_{j} \sigma_{i} \sigma_{i+e_{j}}
$$

where $e_{j}$ is the unit vector in the $j^{\text {th }}$ coordinate direction. Then (4.3) defines a $d$ dimensional Ising model. For simplicity we consider only the case in which $J_{d} \rightarrow 0$ and set $J_{1}=\ldots=J_{d-1}=1$. Then $\beta_{c}=\beta_{c}\left(J_{d}\right)$ is a function of $J_{d}$. As above, we have

Theorem 3. For $J_{d} \geqq 0, m$ is continuous and $\beta_{c}$ is semicontinuous as functions of $J_{d}$, in the single phase region.

Remark. Continuity of $\beta_{c}$ would follow from a uniform lower bound on $d^{2} / d(-\beta)$, for example $v<\infty$.

\section{Trivial vs. Nontrivial Fields}

We give a simple sufficient condition for triviality of the $\phi^{4}$ continuum limit. We parametrize the single phase even $\phi^{4}$ lattice fields by $\lambda(=$ bare charge $), m(=$ physical mass), $\varepsilon(=$ lattice spacing $)$ and $d(=$ space time dimension).

Theorem 4. Suppose that $Z=Z(\lambda, m, \varepsilon, d)$ is continuous and bounded away from zero for $0 \leqq \lambda \leqq \infty, 0 \leqq m \leqq \delta, \varepsilon=1$, and for some $\delta>0$, and some $d \geqq 3$. Then the continuum limit of an even single phase d-dimensional $\phi^{4}$ lattice field with arbitrary charge renormalization has a free two point function.

Proof. The continuum fields are constructed with the arbitrary renormalization $\lambda$ $=\lambda(\varepsilon), 0<m=m(\varepsilon) \leqq$ const, where $\varepsilon_{j} \rightarrow 0$ through a subsequence. Existence of the limit follows from the bounds of $[6,4]$, which establish compactness of the sequence of Schwinger functions. Properties of the $\varepsilon \rightarrow 0$ theory are reduced to properties of the $\varepsilon=1$ theory by a (canonical) scale transformation. $Z$ is invariant under scale transformations, so that

$$
Z(\lambda(\varepsilon), m(\varepsilon), \varepsilon)=Z\left(\varepsilon^{4-d} \lambda(\varepsilon), \varepsilon m(\varepsilon), 1\right) .
$$

There is a similar transformation law for the spectral weight of the two point function. The Fourier transformed two point function at zero momentum has the Herglotz representation [11]

$$
\begin{aligned}
G^{(2) \sim}\left(p_{0}, \boldsymbol{p}=0\right)= & Z\left(\cosh m=\cos p_{0}\right)^{-1} \\
& +\int_{m+0}^{\infty}\left(\cosh a-\cos p_{0}\right)^{-1} \varrho \varrho(a, \lambda, m, \varepsilon, d)
\end{aligned}
$$


for $\varepsilon=1$. Continuity of $Z$ at $m=0$ can be expressed as the following property of the measure $d \varrho$ :

$$
\lim _{\gamma \rightarrow 0} \lim _{m \rightarrow 0} \int_{m^{2}+0}^{\gamma} d \varrho\left(a, \lambda_{1}(m), 1, d\right)=0
$$

for any $\lambda=\lambda_{1}(m)$. We choose $m=\varepsilon m(\varepsilon) \rightarrow 0$ and

$$
\lambda_{1}(\varepsilon m(\varepsilon))=\varepsilon^{4-d} \lambda(\varepsilon) \text {. }
$$

\section{Under scaling, (5.1) transforms into}

$$
\lim _{\gamma \rightarrow 0} \lim _{\varepsilon \rightarrow 0} \int_{m(\varepsilon)^{2}+0}^{\varepsilon^{-2 \gamma}} d \varrho(a, \lambda(\varepsilon), m(\varepsilon), \varepsilon, d)=0,
$$

which implies that limit two point function is free.

\section{References}

1. Baker,G.: Self interacting boson quantum field theory and the thermodynamic limit in $d$ dimensions. J. Math. Phys. 16, 1324-1346 (1975)

2. Coleman,S.: Secret Symmetry: An introduction to spontaneous symmetry breakdown and guage fields. 1973 Erice Summer School of Physics

3. Fisher, M. : Rigorous Inequalities for critical-point correlation exponents. Phys. Rev. 180, 594-600 (1969)

4. Fröhlich,J., Simon,B., Spencer,T.: A new method for the analysis of phase transitions and spontaneous breaking of discrete and continuous symmetries. Phys. Rev. Lett. 36, 804 -806 (1976)

5. Fröhlich,J., Simon,B.: Pure states for general $P(\phi)_{2}$ theories: Construction, regularity and variational equality. Ann. Math., to appear

6. Glimm,J., Jaffe, A.: A remark on the existence of $\phi_{4}^{4}$. Phys. Rev. Lett. 33, 440-442 (1974)

7. Glimm,J., Jaffe, A. : The $\phi_{2}^{4}$ quantum field theory in the single phase region : Differentiability of the mass and bounds on critical exponents. Phys. Rev. D 10, 536-539 (1974)

8. Glimm,J., Jaffe, A.: Three particle structure of $\phi^{4}$ interactions and the scaling limit. Phys. Rev. D 11, $2816-2827(1975)$

9. Glimm,J., Jaffe,A.: Critical problems in quantum fields, International Colloquium on Mathematical Methods of Quantum Field Theory, Marseille, June 1975

10. Glimm,J., Jaffe, A.: Critical exponents and renormalization in the $\phi^{4}$ scaling limit, Conference on quantum dynamics: Models and mathematics. Acta Phys. Austriaca Suppl. XVI (1975)

11. Glimm, J., Jaffe, A.: Particles and scaling for lattice fields and Ising models. Commun. math. Phys. $\mathbf{5 1}, 1-14$ (1976)

12. Rosen,J.: Mass renormalization for lattice $\lambda \phi_{2}^{4}$ fields. Adv. Math. (to appear)

13. Rosen,J.: The Ising limit of $\phi^{4}$ lattice fields. The Rockefeller University (preprint)

14. Schrader, R.: A possible constructive approach to $\phi_{4}^{4}$ I, III. Commun. math. Phys. 49, $131-154$ (1976); 50, 97-102 (1976)

15. Kato,J. : Perturbation theory for linear operators. Berlin-Heidelberg-New York: Springer 1966

16. Glimm,J., Jaffe, A.: A $\lambda \phi^{4}$ Quantum field theory without cutoffs. I. Phys. Rev. 176, 1945-1951 (1968) and Singular perturbations of self adjoint operators. Comm. Pure Appl. Math. 22, 401-414 (1969)

Communicated by R. Haag

Received June 30, 1976 
